\title{
Identification of differentially expressed genes between primary lung cancer and lymph node metastasis via bioinformatic analysis
}

\author{
NAN ZHANG and SHAO-WEI ZHANG
}

\begin{abstract}
Department of Thoracic Surgery, The Fourth Hospital of Hebei Medical University, Shijiazhuang, Hebei 050000, P.R. China
\end{abstract}
Received January 10, 2019; Accepted July 12, 2019

DOI: $10.3892 / 01.2019 .10723$

\begin{abstract}
Lung cancer (LC), with its high morbidity and mortality rates, is one of the most widespread and malignant neoplasms. Mediastinal lymph node metastasis (MLNM) severely affects postoperative survival of patients with LC. Additionally, the molecular mechanisms of LC with MLNM (MM LC) remain not well understood. To identify the key biomarkers in its carcinogenesis and development, the datasets GSE23822 and GSE13213 were obtained from the Gene Expression Omnibus database. The differentially expressed genes (DEGs) were identified, and the Database for Annotation, Visualization and Integrated Discovery was used to perform functional annotations of DEGs. Search Tool for the Retrieval of Interacting Genes and Cytoscape were utilized to obtain the protein-protein interaction (PPI) network, and to analyze the most significant module. Subsequently, a Kaplan-Meier plotter was used to analyze overall survival (OS). Additionally, one co-expression network of the hub genes was obtained from
\end{abstract}

Correspondence to: Mr. Shao-Wei Zhang, Department of Thoracic Surgery, The Fourth Hospital of Hebei Medical University, 12 Jiankang Road, Shijiazhuang, Hebei 050000, P.R. China E-mail:986450106@qq.com

Abbreviations: ACTB, actin $\beta$; ADAM, ADAM metallopeptidase domain; AVP, arginine vasopressin; BiNGO, Biological Networks Gene Oncology tool; BP, biological processes; CC, cellular components; CXCR4, C-X-C motif chemokine receptor 4; DEGs, differentially expressed genes; ERBB2, Erb-b2 receptor tyrosine kinase 2; GEO, Gene Expression Omnibus; GEPIA, Gene Expression Profiling Interactive Analysis; GO, Gene Ontology; ITGB1, integrin subunit $\beta 1$; ITSN2, intersectin 2; KEGG, Kyoto Encyclopedia of Genes and Genomes; LC, lung cancer; MCODE, Molecular Complex Detection; MF, molecular function; MLNM, mediastinal lymph node metastasis; MM LC, lung cancer with mediastinal lymph node metastasis; non-MM LC, lung cancer samples without mediastinal lymph node metastasis; OS, overall survival; PET, positron emission tomography; PPI, protein-protein interaction; RAB5B, RAB5B, member RAS oncogene family; SYNJ2, synaptojanin 2; VEGF-C, vascular endothelial growth factor-C; VEGF-D, vascular endothelial growth factor-D; VEGFR-3, vascular endothelial growth factor receptor-3; VAMP2, vesicle associated membrane protein 2

Key words: LC, PPI, MLNM, DEGs, bioinformatics analysis
cBioPortal. A total of 308 DEGs were identified in the two microarray datasets, which were mainly enriched during cellular processes, including the Gene Ontology terms 'cell', 'catalytic activity', 'molecular function regulator', 'signal transducer activity' and 'binding'. The PPI network was composed of 315 edges and 167 nodes. Its significant module had 11 hub genes, and high expression of actin $\beta$, MYC, arginine vasopressin, vesicle associated membrane protein 2 and integrin subunit $\beta 1$, and low expression of NOTCH1, synaptojanin 2 and intersectin 2 were significantly associated with poor OS. In summary, hub genes and DEGs presented in the present study may help identify underlying targets for diagnostic and therapeutic methods for MM LC.

\section{Introduction}

Lung cancer (LC) is one of the most malignant neoplasms, and increases in its morbidity and mortality rates have made it a main cause of human mortality. In the last 50 years, research has revealed a marked increase in the incidence and mortality rates of $\mathrm{LC}$ (1). The incidence and mortality rates of LC in men ranked first among all malignant tumor groups, and the incidence in women ranked second most common worldwide in 2018 (2). The onset of LC is insidious and it has a poor prognosis. Mediastinal lymph node metastasis (MLNM) during treatment is one of the most important factors affecting the postoperative survival of patients (3).

The MLNM process of LC includes the growth of a primary tumor, angiogenesis and exfoliation of tumor cells, which invade the tissue matrix, and make cancer cells survive in the blood circulation and amass into tiny tumor thrombi (4). Previous studies in China have reported that the 5-year survival rate of patients with LC without MLNM could be $>60 \%$, but was only $15-42 \%$ with MLNM $(5,6)$. Numerous previous studies (7-11) have demonstrated that the pathophysiological process of the development of LC with MLNM (MM LC) is associated with the mutation and abnormal expression of genes, including $\mathrm{C}-\mathrm{X}-\mathrm{C}$ motif chemokine receptor 4 (CXCR4), vascular endothelial growth factor-C (VEGF-C), vascular endothelial growth factor receptor-3 (VEGFR-3), ADAM metallopeptidase domain (ADAM) and vascular endothelial growth factor-D (VEGF-D). In a previous study by Na et al (9) of 46 patients with LC, abnormal expression of CXCR4 in the nucleus was markedly associated with MLNM. In addition, multiple previous studies have suggested that co-expression of 
VEGFR-3 and VEGF-C (7), high expression levels of ADAM family members (8), downregulation of VEGF-D expression (10), and high expression levels of VEGF-C (11) may be involved in the MLNM of LC. These genes may be used as prognostic factors or targets for gene therapy. However, in individualized applications, the diagnostic or therapeutic value of a single gene remains uncertain. Due to the lack of timely detection, dynamic monitoring and effective control of the occurrence of MLNM, the poor survival rate of MM LC still cannot be effectively controlled. Therefore, novel signaling pathways and molecular targets should be investigated and screened to develop novel diagnostic and therapeutic methods.

Microarray technology allows simultaneous analysis of alterations in the expression levels of multiple genes to obtain gene sets that can predict MLNM in LC with high accuracy. Clinical application of these gene sets is expected to maximize the survival period and narrow the surgical range (whether lymph node resection is required) for the benefit of patients. Previously, a number of studies (12-17) have performed bioinformatic analyses to investigate differentially expressed genes (DEGs) in various types of cancer, as well as their roles in different pathways, molecular functions and biological processes. Kikuchi et al (12) used microarray technology to obtain a set of genes which could predict MLNM and drug sensitivity. Li et al (13) applied the same method to identify the significant genes in the carcinogenesis and progression of hepatocellular carcinoma. Furthermore, multiple previous studies have also used microarray technology to obtain gene expression profiles to predict lymph node metastasis of other malignant tumors, including esophageal cancer (14), oral squamous cell carcinoma $(15,16)$ and cervical cancer $(17)$.

Therefore, the purpose of the present study was to download and analyze two expression profiling datasets of human samples from the Gene Expression Omnibus (GEO) database, and to identify DEGs between LC samples without MLNM (non-MM LC) and MM LC samples. Subsequently, Kyoto Encyclopedia of Genes and Genomes (KEGG) pathway and Gene Ontology (GO) analysis were carried out. Protein-protein interaction (PPI) network analysis and co-expression network analyses were used to demonstrate the molecular pathogenesis underlying carcinogenesis and development of MM LC. Overall, 11 hub genes and 308 DEGs, which may be potential molecular targets or biomarkers for MM LC, were identified.

\section{Materials and methods}

Access to public data. The GEO (http://www.ncbi.nlm.nih. gov/geo) is an open platform for storing genetic data (18). In total, two expression profiling datasets [GSE23822 (19) (GPL6947 platform) and GSE13213 (20) (GPL6480 platform)] were obtained from GEO. The GSE23822 dataset contained four non-MM LC samples and four MM LC samples. Similarly, GSE13213 consisted of 22 non-MM LC samples and $22 \mathrm{MM}$ LC samples.

Identification of DEGs using GEO2R. GEO2R (https://www. ncbi.nlm.nih.gov/geo/geo2r/) is an interactive online tool for the identification of DEGs from GEO series (21). GEO2R was used to identify DEGs between MM LC and non-MM LC tissue samples. If one probe set did not have the homologous gene, or if one gene had numerous probe sets, the data were removed. The rules of statistical significance were that $\mathrm{P} \leq 0.01$ and fold change $\geq 1.5$.

Functional annotation of DEGs by KEGG and GO analysis. Database for Annotation, Visualization and Integrated Discovery (DAVID; version 6.8, https://david.ncifcrf.gov/home. jsp), is an online analysis tool suite with the functions of integrated discovery and annotation (22). GO (http://geneontology. org) is widely used in bioinformatics, and covers three aspects of biology; biological process (BP), cellular component (CC) and molecular function (MF) (23). KEGG (https://www. kegg.jp) is one of the most commonly used biological information databases in the world (24). OmicShare (http://www. omicshare.com/tools), an open data analysis platform, was used to perform GO analysis (25). To analyze the biological pathway information of DEGs, the DAVID online tool was implemented. $\mathrm{P}<0.05$ was considered to indicate a statistically significant difference.

Construction of the PPI network and identification of the significant module. Search Tool for the Retrieval of Interacting Genes (http://string.embl.de/), an online open tool, was applied to construct the PPI network of DEGs, and Cytoscape was used to present the network (26). Cytoscape (version 3.6.1) is a free visualization software (27). A confidence score $>0.4$ was considered as the criterion of judgment. Subsequently, the Molecular Complex Detection (28) (MCODE; version 1.5.1; a plug-in of Cytoscape), was used to identify the most important module of the network map. The criteria for MCODE analysis were as follows: i) Degree cut-off $=2$; ii) MCODE scores $>5$; iii) $\max$ depth=100; iv) node score cut-off $=0.2$; and v) $\mathrm{k}$-score=2 (29). Subsequently, following the KEGG and GO analysis using the DAVID database and OmicShare website, functional annotation for genes of these modules was performed.

Analysis and identification of hub genes. The degrees were set (degrees $\geq 10$ ), and the hub genes were excavated. A co-expression network of these hub genes was obtained from cBioPortal (http://www.cbioportal.org) (30). Furthermore, the Biological Networks Gene Oncology tool (BiNGO; version 3.0.3) was used to analyze and visualize the CCs, BPs and MFs of the hub genes (31). The clustering analysis of hub genes was performed using OmicShare (https://www. omicshare.com/tools/Home/Soft/getsoft/type/index) (25). The mean value of amount of gene expression was defined as the cut-off value for the high or low expression level. Additionally, Kaplan-Meier Plotter (http://kmplot.com/analysis/index. php?p=background), an online analysis tool, was utilized to perform survival analysis for the hub genes. The Kaplan Meier plotter (32) is capable of assessing the effect of 54,000 genes on survival in 21 different types of cancer. The largest datasets include breast $(n=6,234)$, ovarian $(n=2,190)$, lung $(n=3,452)$ and gastric $(n=1,440)$ cancer. The miRNA subsystems include $11 \mathrm{k}$ samples from 20 different cancer types. Primary purpose of the tool is a meta-analysis based discovery and validation of survival biomarkers. The P-value was achieved by using a log-rank test. University of California Santa Cruz (UCSC) Xena (https://xena.ucsc.edu/welcome-to-ucsc-xena/) was used to securely analyze and visualize the hub genes in the scope 

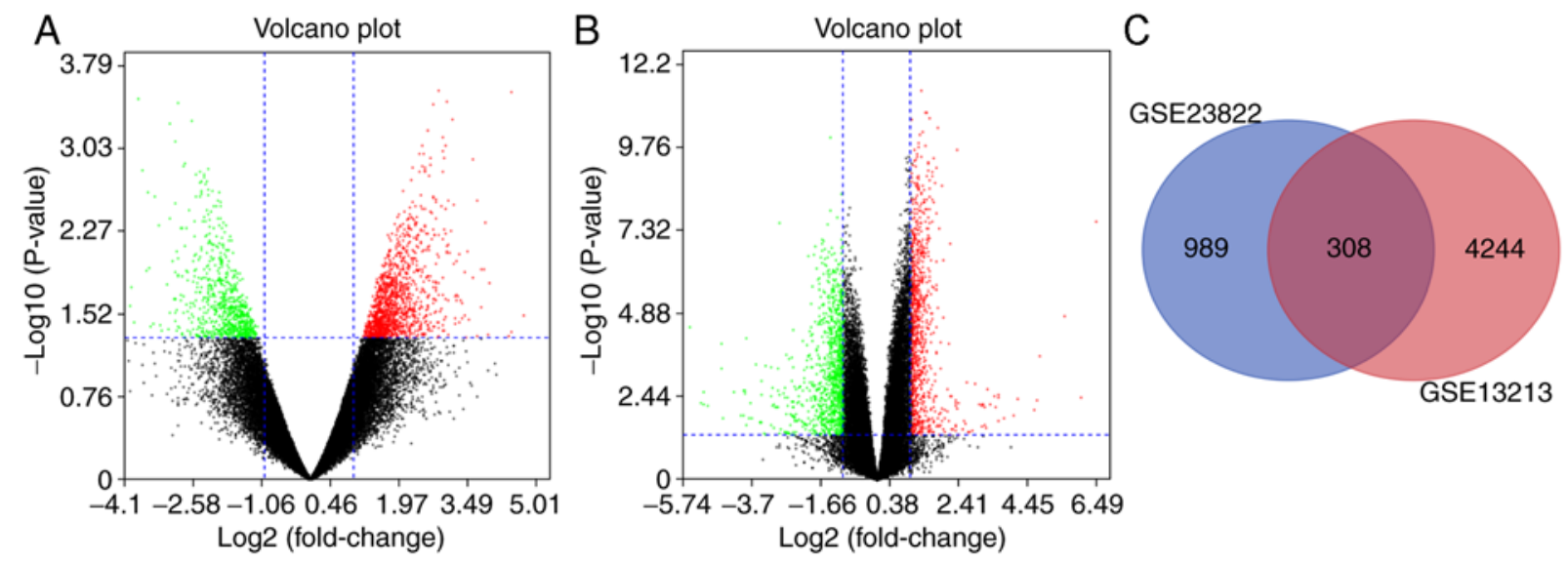

Figure 1. Identification of differently expressed genes between non-MM lung cancer and MM lung cancer tissues. (A) Volcano plot of the difference between non-MM lung cancer and MM lung cancer tissues following analysis of the GSE23822 dataset with GEO2R. (B) Volcano plot presenting the difference between non-MM lung cancer and MM lung cancer tissues following analysis of the GSE13213 dataset with GEO2R. (C) Venn diagram demonstrating that 308 genes were contained in the GSE23822 and GSE13213 datasets simultaneously. MM lung cancer, lung cancer with mediastinal lymph node metastasis; non-MM lung cancer, lung cancer without mediastinal lymph node metastasis.

of public genomic datasets. The expression profiles of actin $\beta$ (ACTB) and integrin subunit $\beta 1$ (ITGB1) were analyzed and visualized using the online database Gene Expression Profiling Interactive Analysis (GEPIA; http://gepia.cancer-pku.cn/).

\section{Results}

Screening of DEGs in MM LC samples. Following analysis of the datasets (GSE23822 and GSE13213) with GEO2R, the difference between MM LC and non-MM LC tissues was presented in volcano plots (Fig. 1A and B). The analyses of GSE23822 and GSE13213 identified 1,297 and 4,552 DEGs, respectively (Fig. 1C). The Venn diagram demonstrated that the commonality between the two datasets included 308 DEGs, including the most upregulated genes [Erb-b2 receptor tyrosine kinase 2 (ERBB2) and ITGB1] and the most downregulated genes [ACTB, RAB5B, member RAS oncogene family (RAB5B) and intersectin 2 (ITSN2)] (data not shown).

Functional annotation of DEGs by KEGG and GO analyses. The results of the GOanalysis demonstrated that variations in the BP were primarily enriched in the GO terms 'cellular process', 'metabolic process', 'response to stimulus', 'biological regulation', 'signaling' and 'localization', 'single-organism process', 'developmental process', 'cellular component organization or biogenesis', and so on. Alterations in CC were mainly enriched in the GO terms 'cell', 'membrane', 'extracellular region' and 'organelle part'. The variations in MF were enriched in the GO terms 'binding', 'catalytic activity', 'molecular transducer activity', 'signal transducer activity' and 'molecular function regulator' (Fig. 2). The enriched GO terms were 'generation of precursor metabolites and energy', 'signal transduction', 'regulation of biological process', 'regulation of cellular process', 'cellular response to stimulus', 'cellular component organization or biogenesis', 'cellular component organization', 'anatomical structure morphogenesis', 'cell communication', 'signaling', 'single organism signaling', 'membrane organization', 'growth', 'cell morphogenesis', 'cellular component morphogenesis', 'immune system process', 'localization', 'transport', 'establishment of localization', and 'vesicle-mediated transport' (Fig. 3A). KEGG analysis revealed that DEGs were enriched in 'ErbB signaling pathway', 'microRNAs in cancer', 'endometrial cancer', 'Jak-STAT signaling pathway', 'non-small cell lung cancer', 'chronic myeloid leukemia', 'hypertrophic cardiomyopathy' and 'Hippo signaling pathway' (Fig. 3B).

Construction of the PPI network and identification of the significant module. Construction of the PPI network and identification of the significant module were performed, and there were 315 edges and 167 nodes in the PPI network (Fig. 4). Furthermore, there were 26 edges and 11 nodes in the significant module (Fig. 5). Using DAVID, KEGG and GO analyses of DEGs involved in the significant module were performed. The results demonstrated that genes in the significant module were enriched in the following categories: 'Cellular process', 'localization', 'signaling', 'cell', 'organelle', 'extracellular region', 'membrane', 'binding', 'molecular function regulator' and 'molecular transducer activity' (Fig. 6). The enriched GO terms were 'generation of precursor metabolites and energy', 'signal transduction', 'regulation of biological processes', 'regulation of cellular processes', 'cellular response to stimulus', 'cellular component organization or biogenesis', 'cellular component organization', 'anatomical structure morphogenesis', and so on (Fig. 7A). The KEGG pathway analysis revealed that genes in the significant module were mainly enriched in 'vasopressin-regulated water reabsorption', 'proteoglycans in cancer', 'thyroid hormone signaling pathway', 'phagosome', 'focal adhesion', 'microRNAs in cancer', 'bladder cancer', 'pathogenic Escherichia coli infection', 'endometrial cancer' and 'shigellosis' (Fig. 7B).

$H u b$ gene selection and analysis. Degrees $\geq 10$ was considered as the criterion of judgment. A total of 11 genes were identified as hub genes using Cytoscape: ITGB1, MYC, ERBB2, NOTCH1, ACTB, RAB5B, arginine vasopressin (AVP), synaptojanin 2 (SYNJ2), ITSN2, SH3 domain containing GRB2 like 2 endophilin A1 and vesicle associated membrane 


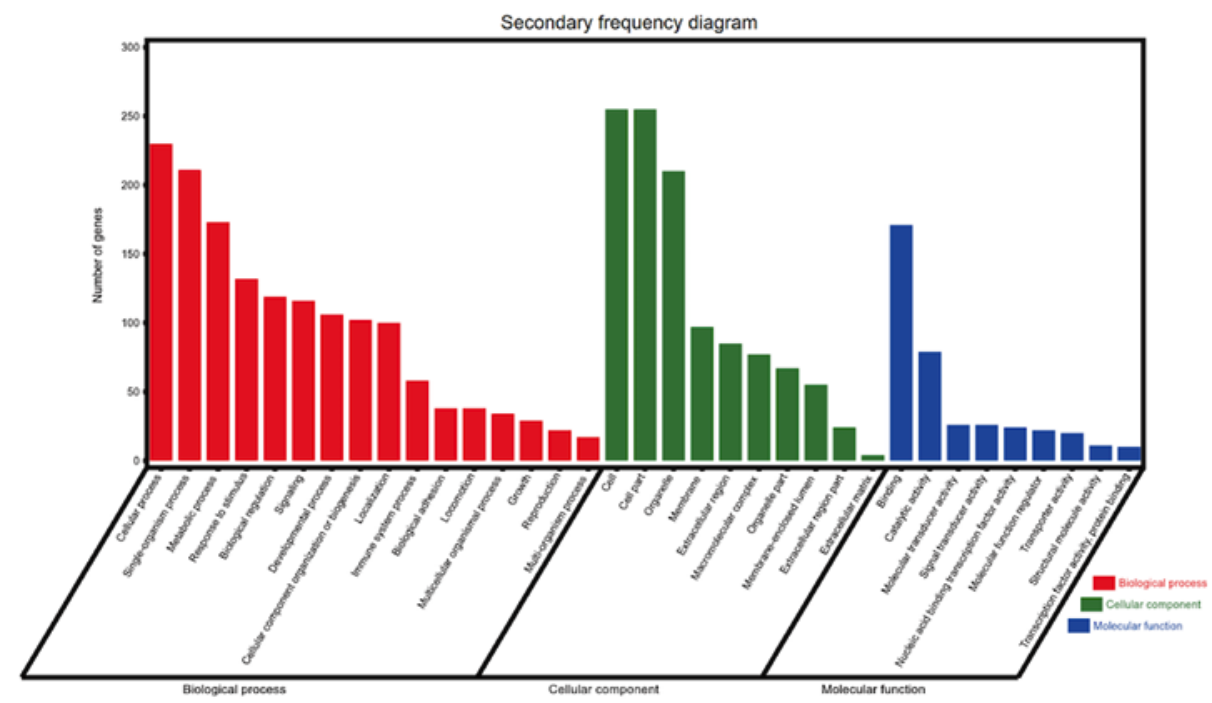

Figure 2. GO enrichment analysis of differentially expressed genes in the form of a secondary frequency diagram. GO, Gene Ontology.

A

Anatomical structure formation involved in morphogenesis
Cellular component organization
Lecalization
Establishment of localization
Cell morphogenesis
Cellular component morphogenesis
Cellular component assembly
Cell differentiation

B

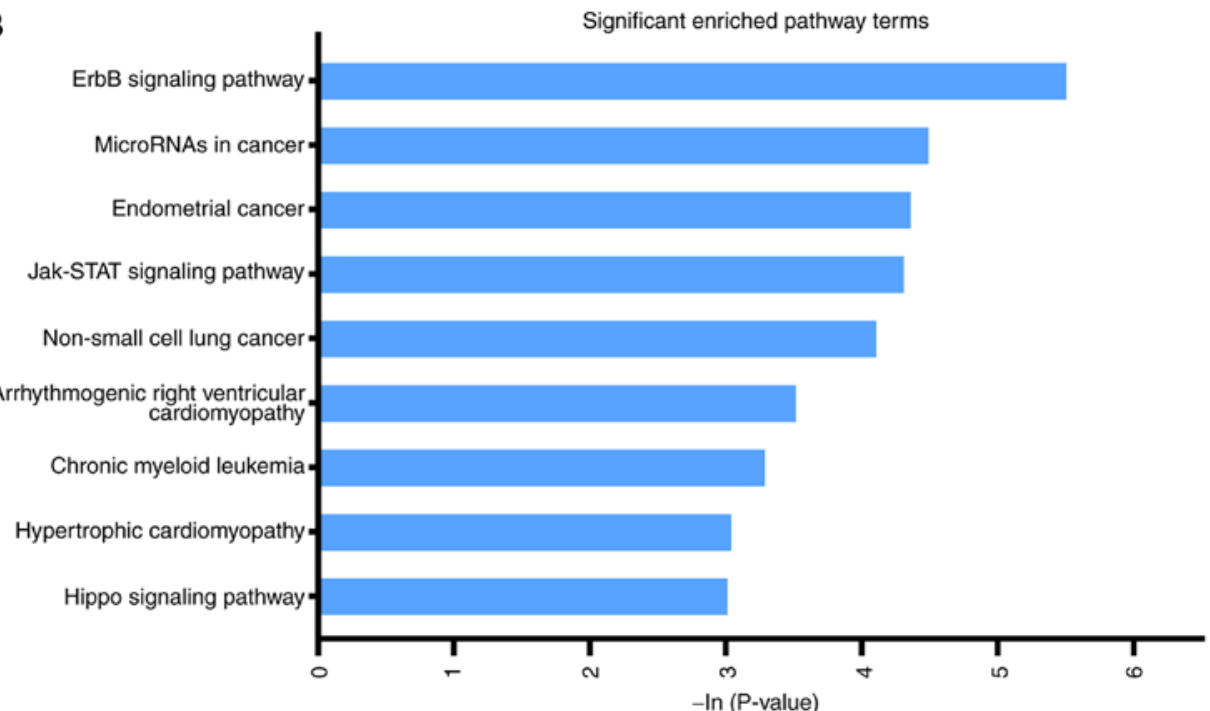

Figure 3. GO and KEGG enrichment analysis of differently expressed genes. (A) GO enrichment analysis of DEGs in the form of an enrichment factor diagram. (B) Kyoto Encyclopedia of Genes and Genomes pathway analysis of DEGs. DEGs, differentially expressed genes; ErbB, Erb-b2 receptor tyrosine kinase; GO, Gene Ontology; Jak, Janus kinase. 


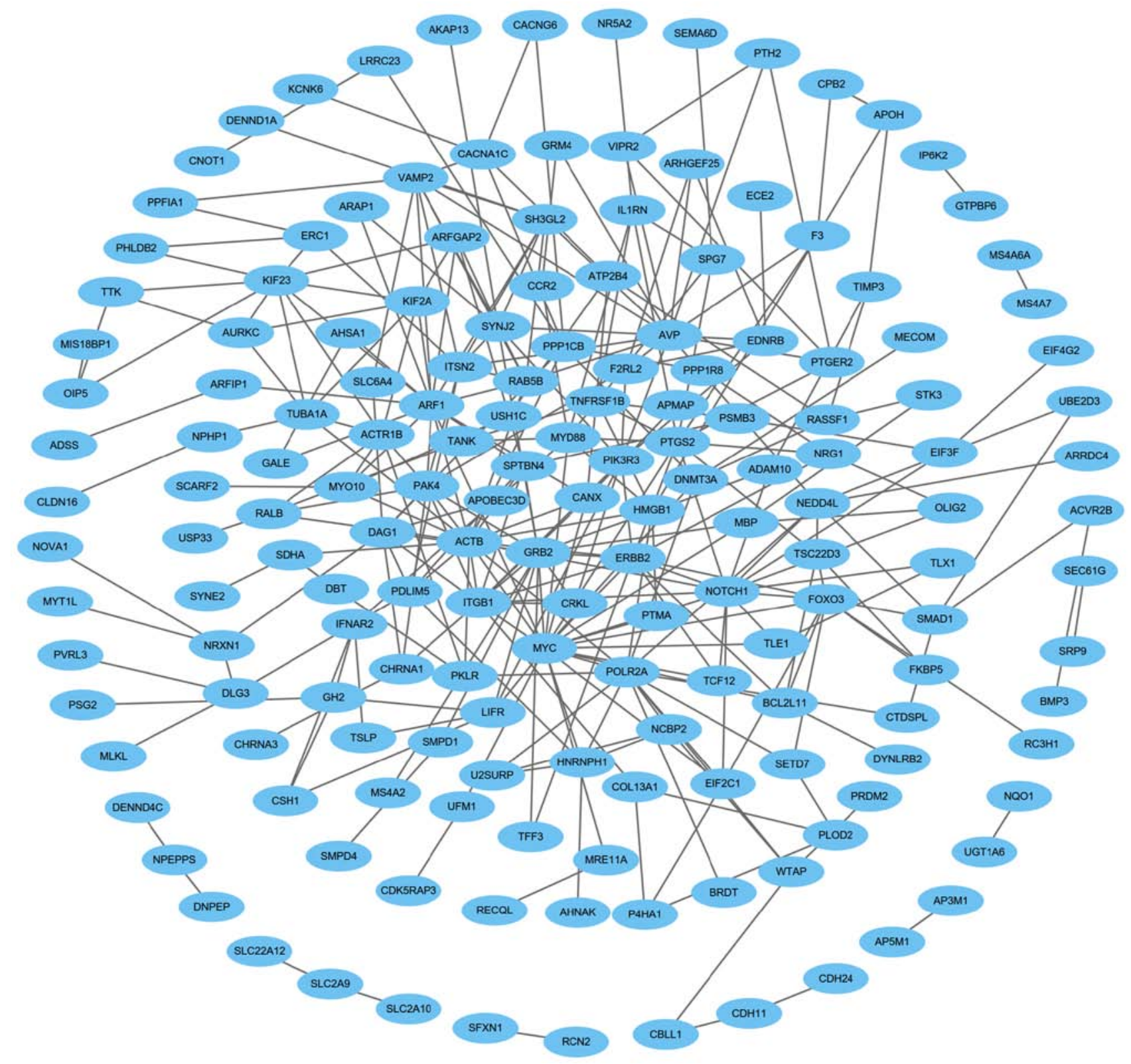

Figure 4. Protein-protein interaction network of differentially expressed genes constructed using Cytoscape.

protein 2 (VAMP2; Table I). A co-expression network of these significant genes was obtained using cBioPortal (Fig. 8). The $\mathrm{BP}, \mathrm{CC}$ and $\mathrm{MF}$ analyses by BiNGO for these genes supported the results of the GO analysis (Figs. S1-S3). The results of the $\mathrm{BiNGO}$ analysis demonstrated that variations in the BP were also mainly enriched for the 'cellular process' term (Fig. S1). Changes in CC were also enriched in cell, membrane ('plasma membrane' and 'plasma membrane part') and organelle terms (Fig. S2). Additionally, the variations in MF were enriched in binding ('kinesin binding', 'nitric-oxide synthase binding' and 'Hsp90 protein binding'), 'molecular transducer activity' (Fig. S3).

Hierarchical clustering revealed that the hub genes could differentiate the MM LC samples from the non-MM LC samples (Fig. 9). Subsequently, a Kaplan-Meier plotter was used to perform overall survival (OS) analysis (Figs. 10 and 11). The samples for OS analysis, derived from the Kaplan-Meier plotter, were different from those used in the analysis of DEGs. Patients with LC with genomic alterations in high expression of ITGB1 (Fig. 10A), high expression of MYC (Fig. 10B), low expression of NOTCH1 (Fig. 10D), high expression of ACTB (Fig. 10E), high expression of AVP (Fig. 11B), low expression of SYNJ2 (Fig. 11C), low expression of ITSN2 (Fig. 11D) and high expression of VAMP2, exhibited poorer OS. However, via the GEPIA, the expression of ACTB (Fig. 10F), ERBB2 (Fig. 10C) RAB5B (Fig. 11A) and SH3GL2 (Fig. 11E), were not associated with OS. In the UCSC Xena analysis, hierarchical clustering revealed that these hub genes could differentiate the patients with LC from the normal patients (Fig. 12A). Among the hub genes, ACTB and ITGB1 had the highest score of 6.415 , suggesting that they may serve important roles in the occurrence or development of MM LC. Using the database of the Kaplan-Meier plotter, the present study identified that high expression of ACTB was associated with poor OS in patients with LE $(\mathrm{P}<0.001)$. Additionally, high expression of ITGB1 was associated with worse OS $(\mathrm{P}=0.024)$. 


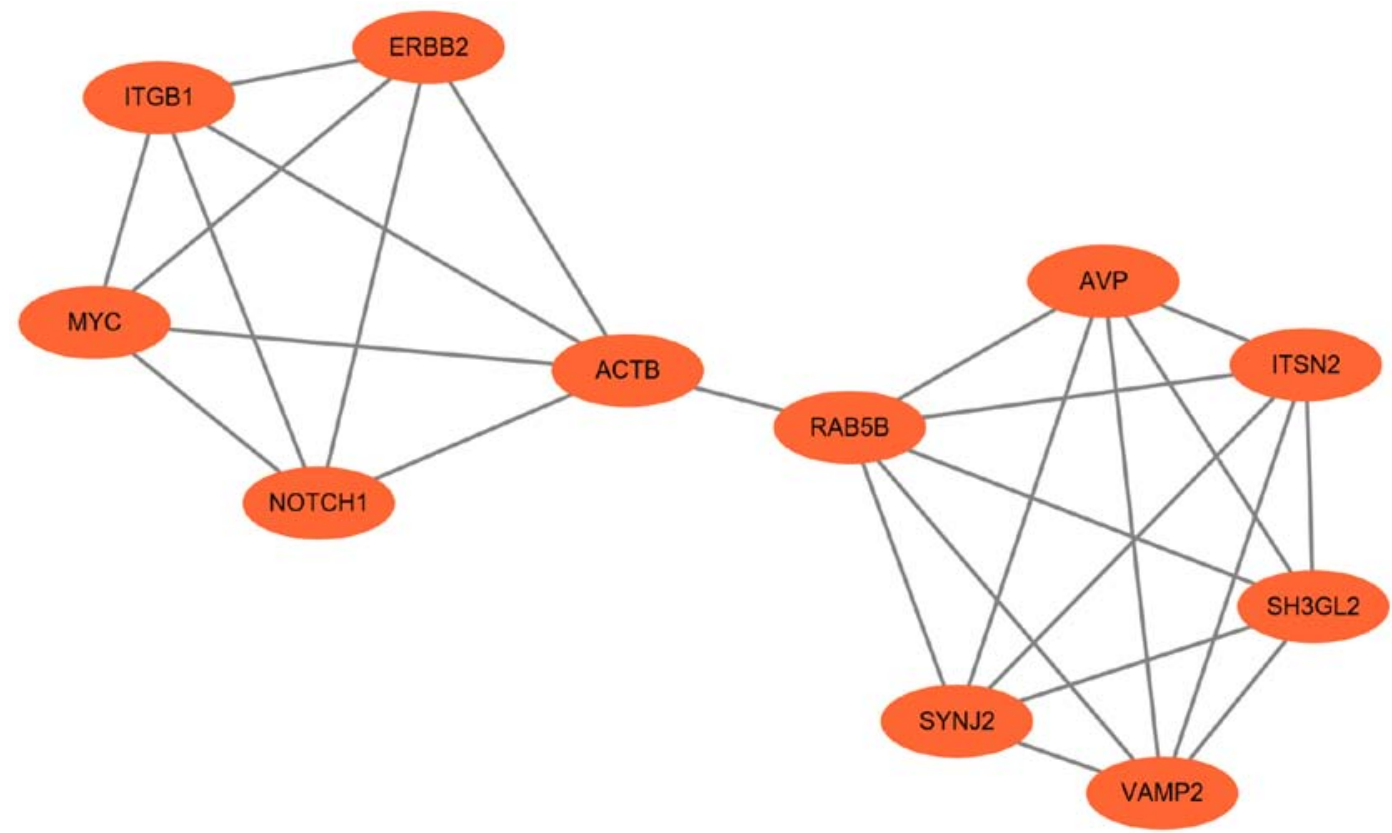

Figure 5. Significant module obtained from the protein-protein interaction network of differentially expressed genes using Molecular Complex Detection. The significant module included 11 nodes and 26 edges. ACTB, actin $\beta$; AVP, arginine vasopressin; ERBB2, ERb-b2 receptor tyrosine kinase 2; ITGB1, integrin subunit $\beta 1$; ITSN2, intersectin 2; RAB5B, RAB5B, member RAS oncogene family; SH3GL2, SH3 domain containing GRB2 like 2, endophilin A1; SYNJ2, synaptojanin 2; VAMP2, vesicle associated membrane protein 2.

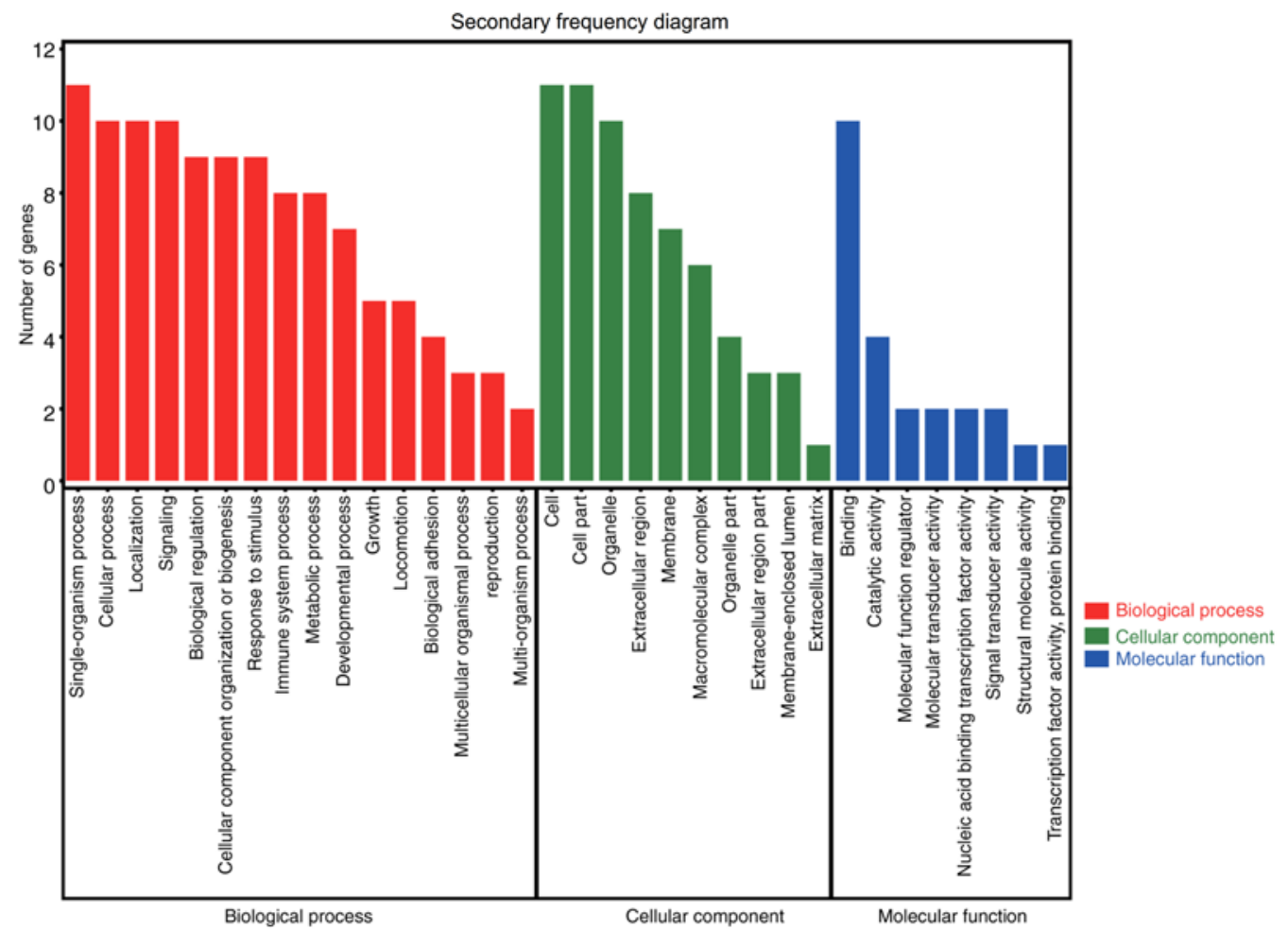

Figure 6. GO enrichment analyses of hub genes in the form of a secondary frequency diagram. GO, Gene Ontology.

The expression profiles of ACTB and ITGB1 in human tissues were visualized using GEPIA. The present study revealed that ACTB and ITGB1 exhibited lower expression levels in lung squamous cell carcinoma compared with the matched normal samples, but no statistically significant difference was identified (P>0.05; Fig. 12B and C).

\section{Discussion}

LC causes the highest cancer-associated mortality rate in China and the majority of countries worldwide. The most common pathological type of LC is non-small-cell LC, which accounts for $80-85 \%$ of LC cases (33). Optimization 
A
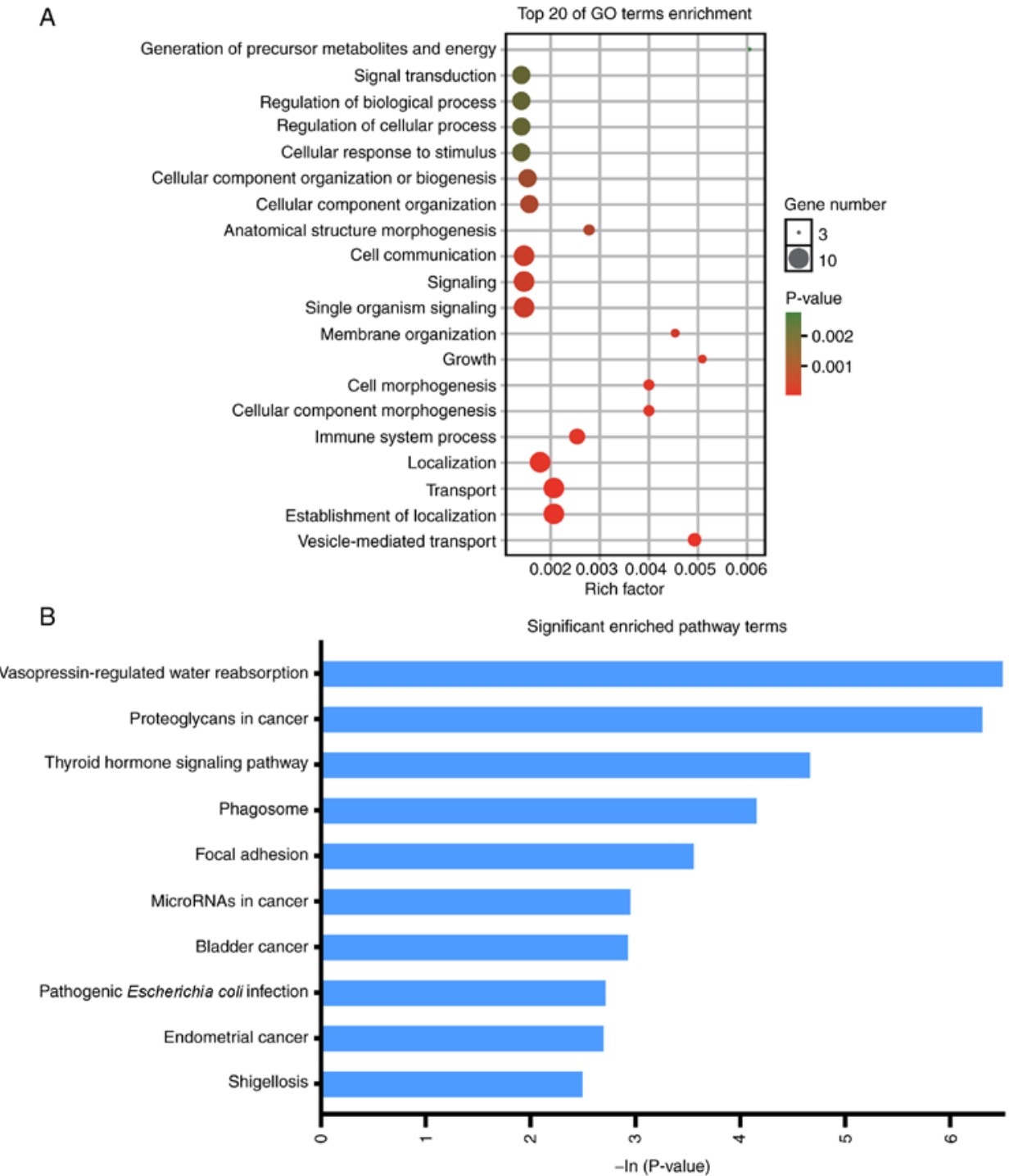

Figure 7. GO and KEGG enrichment analysis of hub genes. (A) GO enrichment analyses of hub genes in the form of an enrichment factor diagram. (B) Kyoto Encyclopedia of Genes and Genomes pathway analysis of hub genes. GO, Gene Ontology.

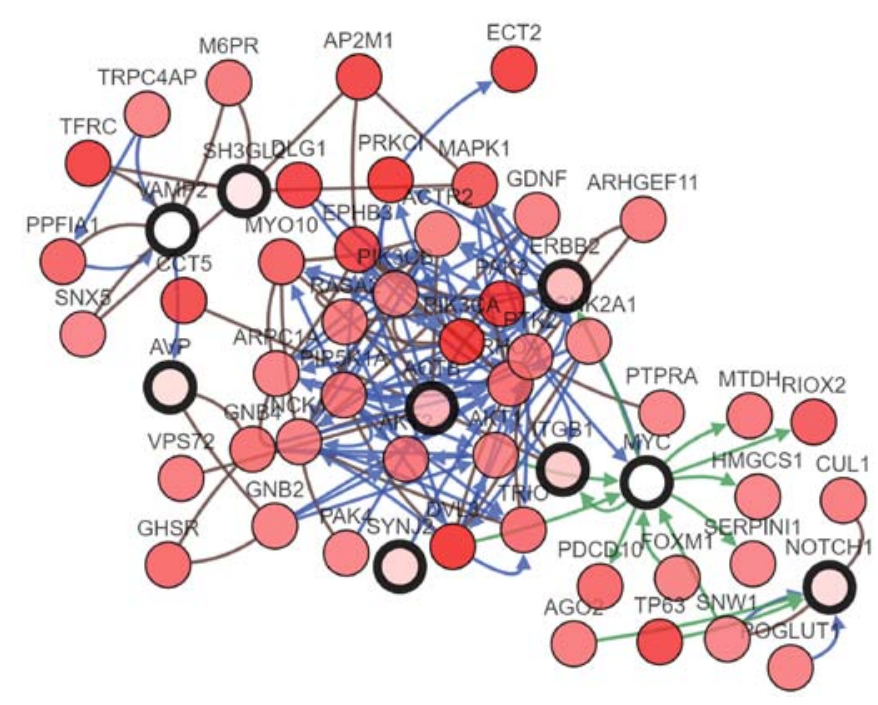

Figure 8. Hub genes and their co-expressed genes were analyzed using cBioPortal. Nodes with a bold black outline represent hub genes. Nodes with a thin black outline represent co-expressed genes. The blue arrows represent 'Controls state change of'. The green arrows represent 'Controls expression of'. The grey arrows represent 'In complex with'. of LC treatment strategies depends on accurate pathological staging and International Association for the Study of Lung Cancer pathological TNM staging (34). In clinical practice, patients with stages 0 , I, II and IIIa may benefit from surgery. For patients with MLNM, neoadjuvant chemotherapy could prolong their postoperative survival (35). For patients without surgical indications, the definite diagnosis of mediastinal lymph node staging could effectively reduce the irradiation area of the radiation target area, which may reduce the incidence of radioactive lung injury. Therefore, if MLNM can clearly defined prior to treatment, it is of great significance to develop appropriate treatment schemes to improve the prognosis of patients.

Currently, there are numerous clinical diagnostic methods for MLNM of LC, including computed tomography (CT), positron emission tomography (PET) and PET-CT. CT is the most widely used diagnostic method for MLNM. A previous study demonstrated that imaging methods continue to have limitations in the evaluation of MLNM, and some patients cannot be clearly diagnosed or are misdiagnosed (36). Diagnosis of MLNM using $\mathrm{CT}$ is mainly based on the size of lymph nodes. The larger the 


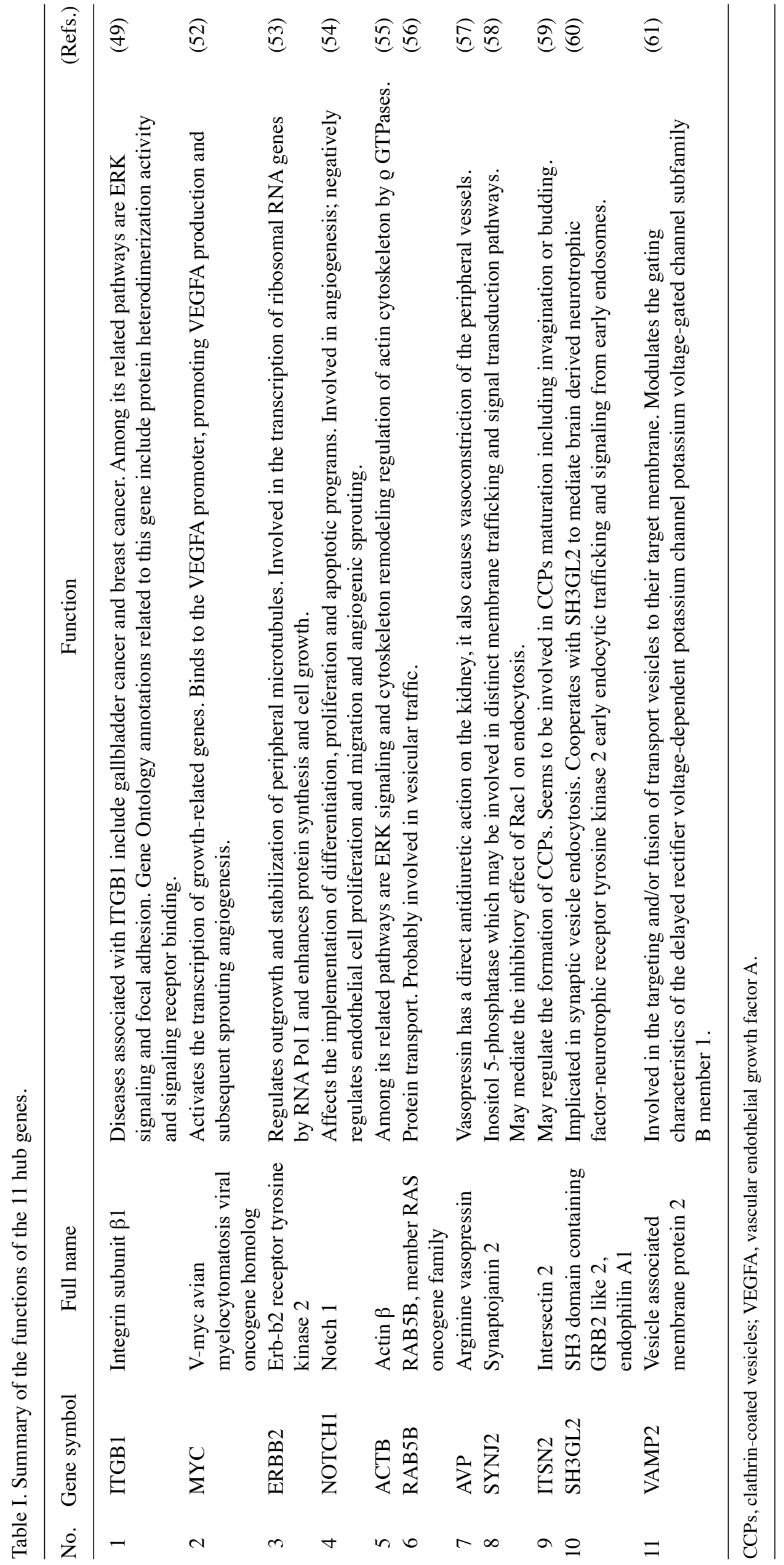


A

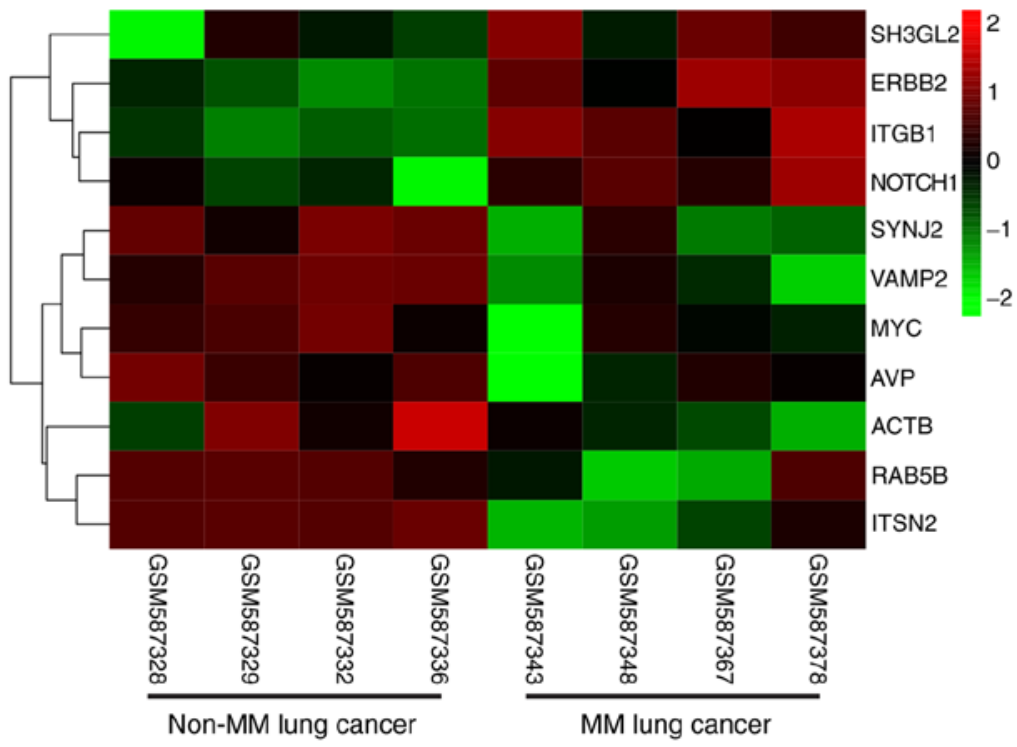

B

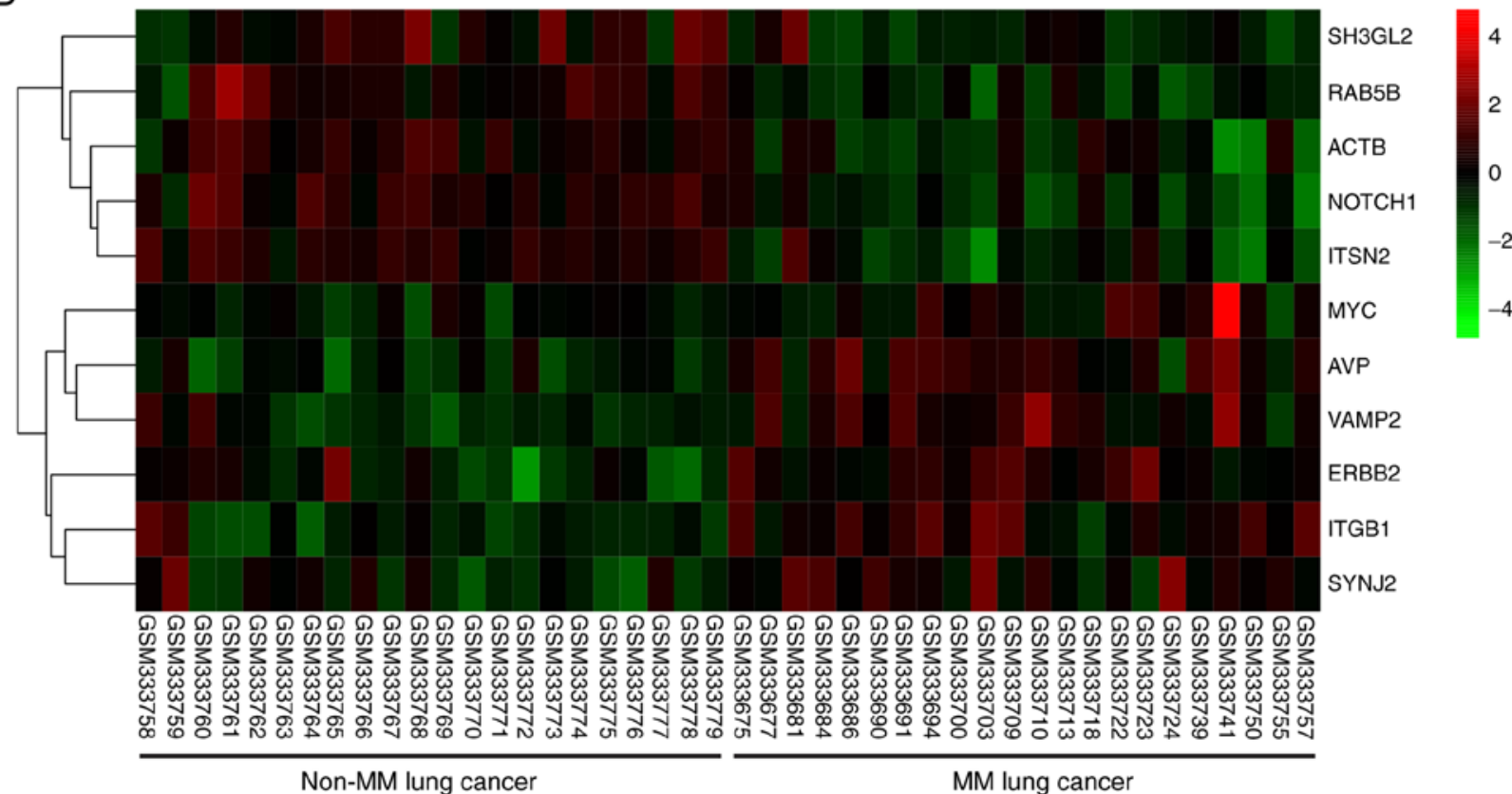

Figure 9. Hierarchical clustering reveals that the hub genes may differentiate the MM lung cancer samples from the non-MM lung cancer samples. This was conducted in the (A) GSE23822 and (B) GSE13213 datasets. Upregulation of genes is marked in red and downregulation of genes is marked in green. ACTB, actin $\beta$; AVP, arginine vasopressin; ERBB2, ERb-b2 receptor tyrosine kinase 2; ITGB1, integrin subunit $\beta 1$; ITSN2, intersectin 2; MM lung cancer, lung cancer with mediastinal lymph node metastasis; non-MM lung cancer, lung cancer without mediastinal lymph node metastasis; RAB5B, RAB5B, member RAS oncogene family; SH3GL2, SH3 domain containing GRB2 like 2, endophilin A1; SYNJ2, synaptojanin 2; VAMP2, vesicle associated membrane protein 2.

lymph nodes, the higher the metastasis rate. The average size of the short diameter of normal lymph nodes depends on the region in which they are located. McLoud et al (37) evaluated 443 lymph nodes in 143 patients using CT. The sensitivity to lymph nodes of various regions was $17-78 \%$, and the specificity was $72-94 \%$. The previous study demonstrated that there were some differences in the sensitivity and specificity of diagnosis using CT to evaluate mediastinal lymph nodes in different subgroups (31). In addition, PET is superior to CT in the diagnosis of MLNM, since PET considers not only lymph node size, but also lymph node metabolism information. A meta-analysis by Toloza et al (38) of PET examination of 1,045 patients in 18 studies manifested that PET was more accurate than $\mathrm{CT}$, and the total sensitivity and specificity were 0.84 (95\% CI, 0.78-0.89) and 0.89 (95\% CI, 0.83-0.93), respectively. However, the diagnostic method of PET has relatively high rates of false negatives and false positives (39). Furthermore, PET-CT effectively combines the technical advantages of CT and PET, which could significantly improve the accuracy of preoperative diagnosis of MLNM in LC. However, several previous studies have reported that PET-CT has high specificity and low sensitivity $(40,41)$. The sensitivity of PET-CT in preoperative assessment of MLNM of lung adenocarcinoma is too low, and further surgical staging is required for patients with LC without MLNM detected by PET-CT.

To overcome the limitations of imaging evaluation of MLNM, previous studies have attempted to identify molecular biomarkers of MM LC (7-10). During the past decades, bioinformatics technology has been generally used to screen 

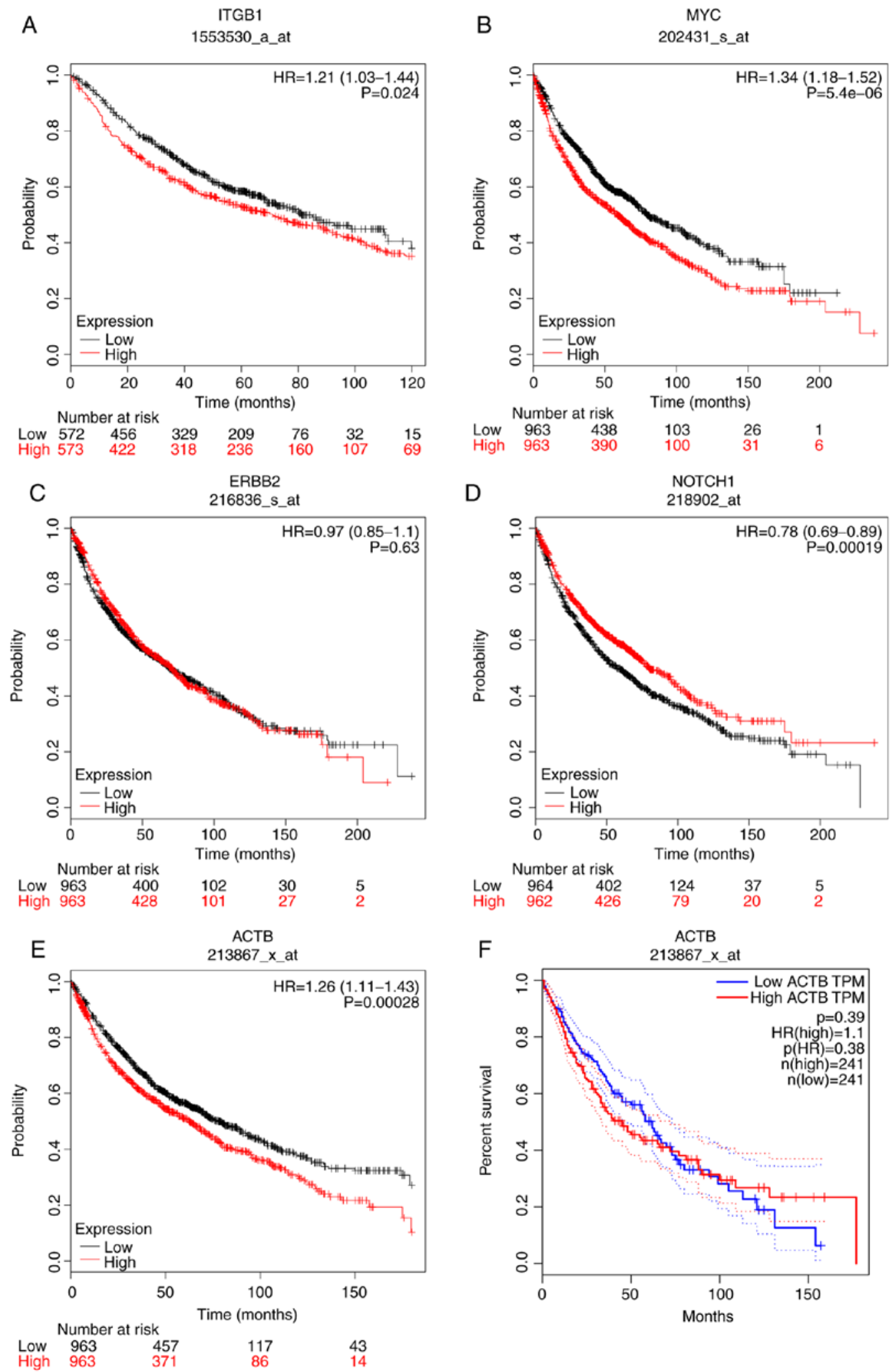

Figure 10. Overall survival analysis of five hub genes (A) Overall survival analysis of ITGB1, (B) MYC, (C) ERBB2, (D) NOTCH1, (E) ACTB, using a Kaplan-Meier plotter online platform. (F) Overall survival analysis of ACTB using GEPIA. $P<0.05$ was considered to indicate a statistically significant difference. ACTB, actin $\beta$; ERBB2, ERb-b2 receptor tyrosine kinase 2; HR, hazard ratio; ITGB1, integrin subunit $\beta 1$; TPM, transcripts per million.

potential genetic targets of diseases, which assisted the authentication of DEGs and underlying pathways associated with the occurrence and recurrence of diseases.

Following analysis of the two microarray datasets in the present study, DEGs between non-MM LC and MM LC were identified. A total of 308 DEGs were contained in the two datasets simultaneously. From the KEGG and GO analyses, the interactions of the DEGs were explored. The DEGs were mainly enriched in the GO terms 'cellular process', 'signaling', 'cell', 'organelle', 'binding', 'molecular transducer activity' 


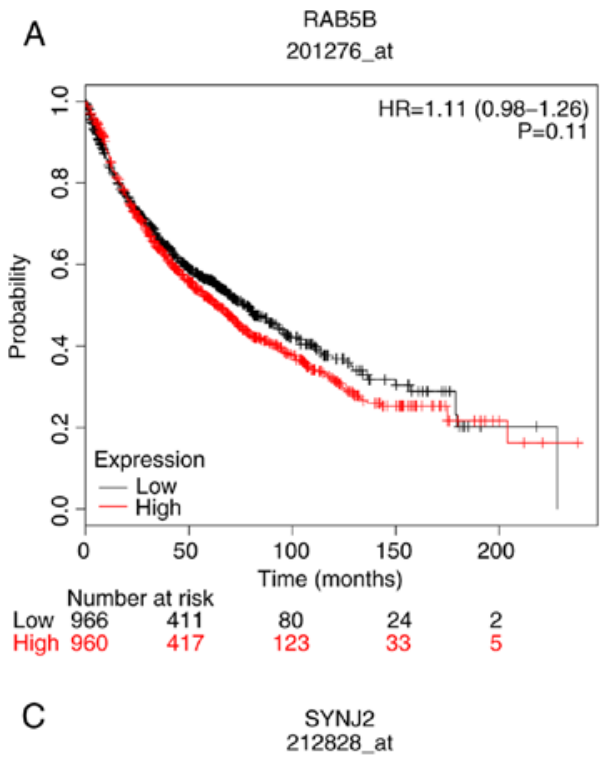

B

AVP

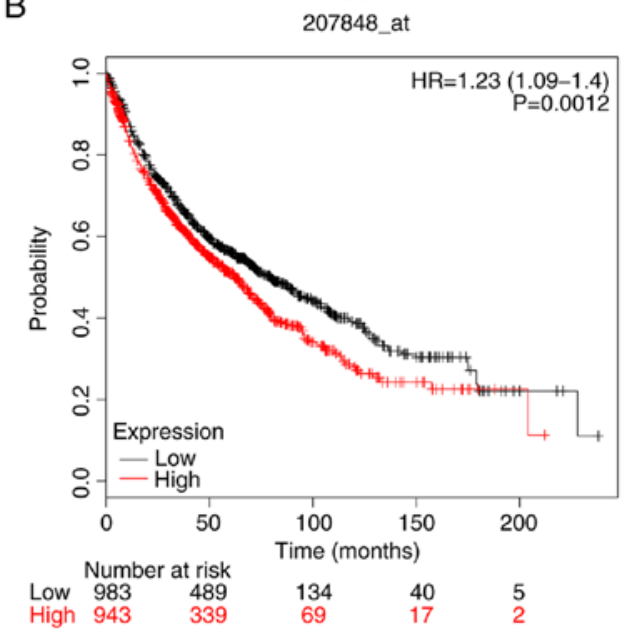

D
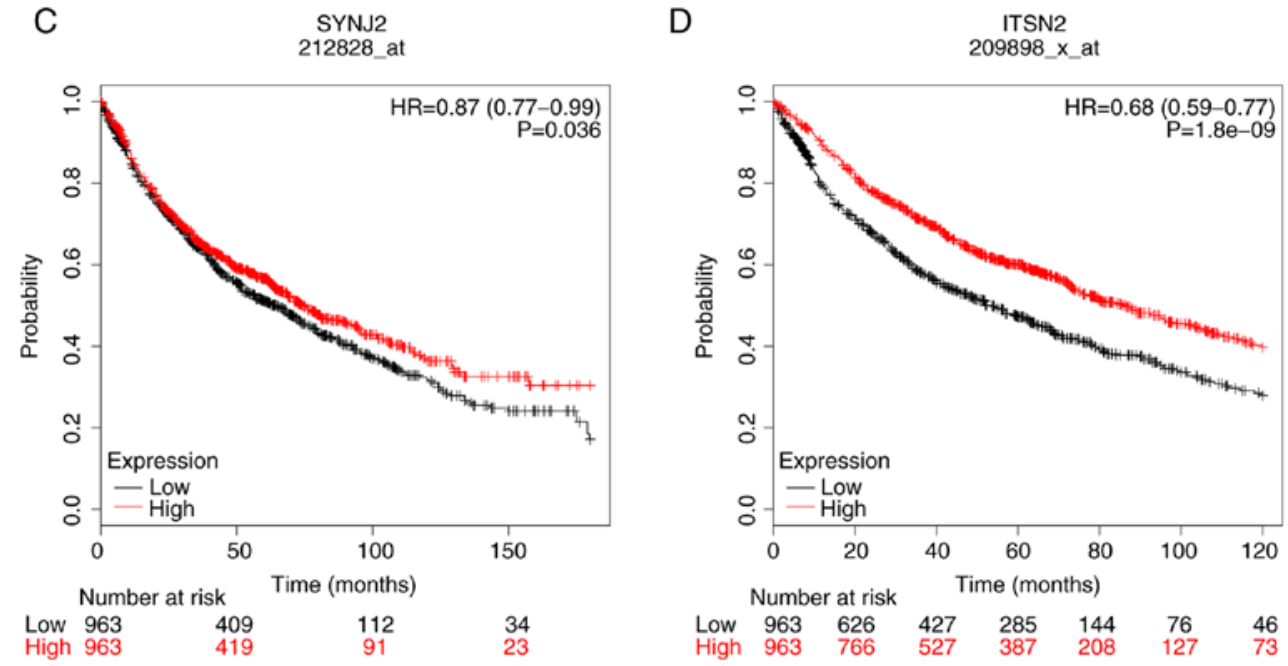

$\mathrm{E}$

SH3GL2

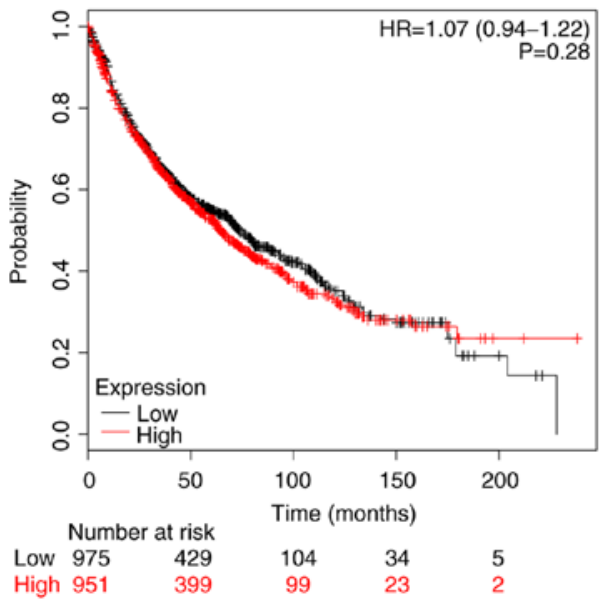

$\mathrm{F}$

VAMP2

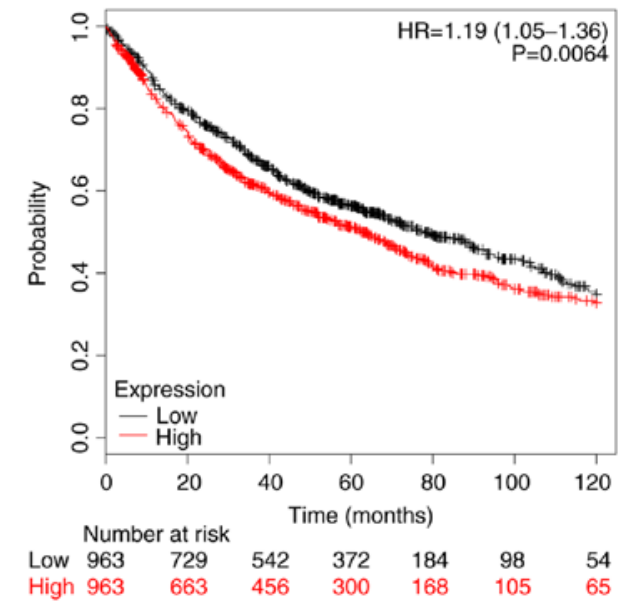

Figure 11. Overall survival analysis of the other six hub genes using a Kaplan-Meier plotter online platform. Overall survival analysis of (A) RAB5B, (B) AVP, (C) SYNJ2, (D) ITSN2, (E) SH3GL2 and (F) VAMP2. P $<0.05$ was considered to indicate a statistically significant difference. AVP, arginine vasopressin; HR, hazard ratio; ITSN2, intersectin 2; RAB5B, RAB5B, member RAS oncogene family; SH3GL2, SH3 domain containing GRB2 like 2, endophilin A1; SYNJ2, synaptojanin 2; VAMP2, vesicle associated membrane protein 2 .

and 'molecular function regulator'. Among the hub genes, ACTB and ITGB1 exhibited the highest score of 6.415 via the MCODE analysis, suggesting that they may serve important roles in the occurrence or development of MM LC.
Actin $\beta$, encoded by the ACTB gene, is widely present in non-muscle cells in the form of a ball or fiber, and participates in the construction of the cytoskeleton and cell movement. As a downstream regulatory protein, actin $\beta$ has the function 


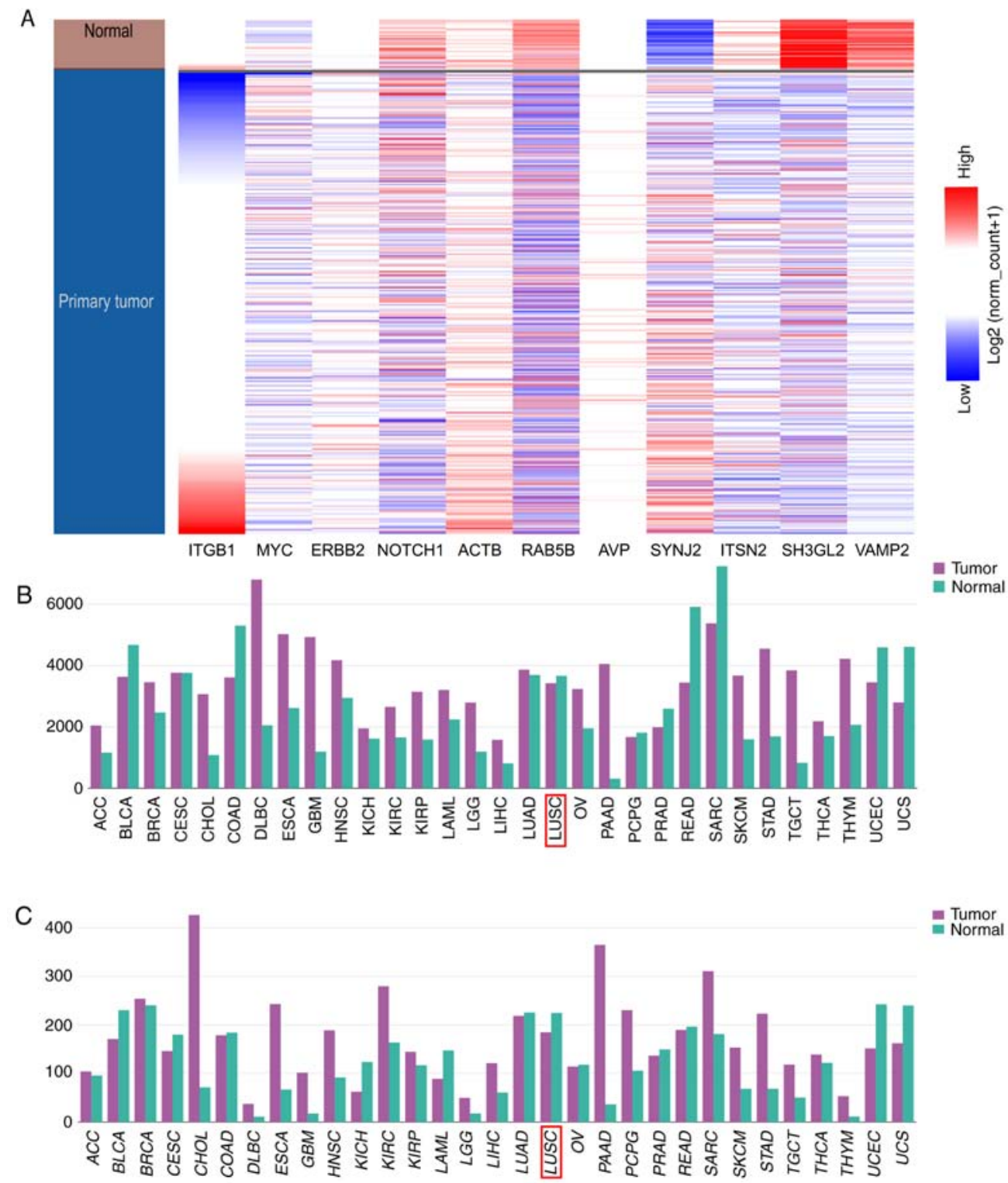

Figure 12. Expression analysis of hub genes. (A) Hierarchical clustering of hub genes using University of California Santa Cruz Xena. Samples next to the brown bar are normal samples and the samples next to the blue bar are lung cancer samples. Upregulation of genes is marked in red. Downregulation of genes is marked in blue. (B) Expression profile of actin $\beta$ in human tumor and normal tissues as obtained using GEPIA. (C) Expression profile of integrin subunit $\beta 1$ in human tissues as obtained using GEPIA. GEPIA, Gene Expression Profiling Interactive Analysis; LUSC, lung squamous cell carcinoma.

of maintaining normal cell migration, growth, differentiation and signal transduction (42). Therefore, it may also be involved in the occurrence mechanism of vascular remodeling. Numerous previous studies (43-45) have demonstrated a close association between ACTB and the occurrence of tumors. Lim et al (43) reported that the mutation of ACTB may cause pilocytic astrocytoma in their clinical experience. Furthermore, the fusions of ACTB and glioma-associated oncogene homolog 1 (GLI1) were regarded as a specific genetic abnormality, which could result in a distinctive type of actin-positive, perivascular myoid tumors, known as 'pericytoma with the $\mathrm{t}(7 ; 12)$ translocation' (44). Furthermore, Castro et al (45) reported that the extremely unusual translocation $\mathrm{t}(7 ; 12)$ may lead to the gene fusion of ACTB and GLI1, which may induce an infrequent gastric tumor derived from the pyloric wall of the stomach. The results of the present study revealed that the expression levels of ACTB in MM LC were downregulated; therefore, the production of actin $\beta$ was reduced, which may lead to abnormal growth, differentiation and exfoliation of LC cells. The detached cancer cells first enter the mediastinal lymph nodes to form a cancer embolus. According to the Kaplan-Meier survival analysis, patients with low expression levels of ACTB had a good prognosis $(\mathrm{P}<0.05)$. However, the expression levels of ACTB had no significant effects on the prognosis based on the survival analysis of GEPIA ( $\mathrm{P}>0.05)$. The influence of ACTB expression on the prognosis was undefined; therefore, more data are required to verify the suggested effect.

ITGB1 is a member of the integrin family of proteins. Integrin family proteins are involved in the regulation of cell adhesion and recognition processes, including hemostasis, embryogenesis, immune response, tissue repair and tumor cell metastasis (46). Yan et al (47) reported that the expression levels of ITGB1 were associated with OS and metastasis 
in patients with aggressive breast cancer. Wang et al (48) reported that linc-ITGB1 promoted the invasion and migration of gallbladder cancer cells by activating epithelial-mesenchymal transition, and knockout of ITGB1 significantly inhibited the metastasis and invasion of gallbladder cancer cells. Klahan et al (49) knocked out ITGB1 in breast cancer cells and revealed that calcium influx decreased, resulting in a significant decrease in the invasion and metastasis of triple-negative breast cancer cells. Wang et al (50) reported that ITGB1 serves important roles in the occurrence and metastasis of LC. The findings of Qin et al (51) suggested that microRNA-134 suppresses migration and invasion of non-small cell LC by targeting ITGB1. The present study demonstrated that the expression levels of ITGB1 were upregulated in MM LC. According to the OS analysis, patients with high expression levels of ITGB1 had a poor prognosis. The reason for this may be that high ITGB1 expression induces the occurrence of MLNM, which could invade the systemic organs along the lymphatic duct, causing systemic organ failure and a shorter lifespan. Based on the aforementioned results, the alterations in the expression levels of ITGB1 may be a molecular mechanism for stimulating the metastasis and invasion of LC cells to the mediastinal lymph node. However, currently, studies regarding IGTB1 are rare, so more efforts should be made in the future.

There are some limitations of the present study. First, the results of the present study are based on bioinformatics analysis only. Therefore, they require laboratory work to be verified using a large set of samples of patients with LC. Currently, it is difficult to obtain the ethical approval documents and informed consent. In the next stage of research, ethical approval and informed consent will be obtained to perform verification of the results of the present study in humans and animals.

In conclusion, the present study aimed to identify DEGs which may be involved in the occurrence or development of LC. Finally, 308 DEGs and 11 hub genes were identified by comparisons between MM LC and non-MM LC samples, which could be used as diagnostic and therapeutic biomarkers for MM LC. The present study provided novel insight for the diagnosis and treatment of MM LC. The results suggested that data mining and integration could be a promising tool to predict biomarkers of malignant tumors. However, the present study is only a preliminary report, and the number of samples in the present study was limited. Since cancer biomarkers only have meaning if they are integrated with clinical data, further experiments should be conducted to confirm the conclusions of the present study.

\section{Acknowledgements}

Not applicable.

\section{Funding}

No funding was received.

\section{Availability of data and materials}

The datasets used and/or analyzed during the current study are available from the corresponding author on reasonable request.

\section{Authors' contributions}

$\mathrm{NZ}$ performed the experiment, and was a major contributor in writing and submitting the manuscript. SWZ made substantial contributions to the conception and design of the study, as well as the acquisition, analysis and interpretation of the data, and also designed the draft of the research process. NZ was involved in critically revising the manuscript for intellectual content. All authors read and approved the final manuscript.

\section{Ethics approval and consent to participate}

Not applicable.

\section{Patient consent for publication}

Not applicable

\section{Competing interests}

The authors declare that they have no competing interests.

\section{References}

1. Lopez-Pastorini A, Riedel R, Koryllos A, Beckers F, Ludwig C and Stoelben E: The impact of preoperative elevated serum C-reactive protein on postoperative morbidity and mortality after anatomic resection for lung cancer. Lung Cancer 109: 68-73, 2017.

2. Bray F, Ferlay J, Soerjomataram I, Siegel RL, Torre LA and Jemal A: Global cancer statistics 2018: GLOBOCAN estimates of incidence and mortality worldwide for 36 cancers in 185 countries. CA Cancer J Clin 68: 394-424, 2018.

3. Isaka M, Kojima H, Takahashi S, Omae $\mathrm{K}$ and Ohde $\mathrm{Y}$ : Risk factors for local recurrence after lobectomy and lymph node dissection in patients with non-small cell lung cancer: Implications for adjuvant therapy. Lung Cancer 115: 28-33, 2018.

4. Yeh YC, Kadota K, Nitadori J, Sima CS, Rizk NP, Jones DR, Travis WD and Adusumilli PS: International association for the study of lung cancer/American thoracic society/European respiratory society classification predicts occult lymph node metastasis in clinically mediastinal node-negative lung adenocarcinoma. Eur J Cardiothorac Surg 49: e9-e15, 2016.

5. Wang S, Zhou W, Zhang H, Zhao M and Chen X: Analysis of predictive factors for postoperative survival for non small cell lung carcinoma patients with unexpected mediastinal lymph nodes metastasis. Thorac Cardiovasc Surg 62: 126-132, 2014.

6. Ma K, Chang D, He B, Gong M, Tian F, Hu X, Ji Z and Wang T: Radical systematic mediastinal lymphadenectomy versus mediastinal lymph node sampling in patients with clinical stage IA and pathological stage T1 non-small cell lung cancer. J Cancer Res Clin Oncol 134: 1289-1295, 2008.

7. SaintignyP,KambouchnerM,LyM,Gomes N,Sainte-CatherineO, Vassy R, Czernichow S, Letoumelin P, Breau JL, Bernaudin JF and Kraemer M: Vascular endothelial growth factor-C and its receptor VEGFR-3 in non-small-cell lung cancer: Concurrent expression in cancer cells from primary tumour and metastatic lymph node. Lung Cancer 58: 205-213, 2007.

8. Ohtsuka T, Shiomi T, Shimoda M, Kodama T, Amour A, Murphy G, Ohuchi E, Kobayashi K and Okada Y: ADAM28 is overexpressed in human non-small cell lung carcinomas and correlates with cell proliferation and lymph node metastasis. Int J Cancer 118: 263-273, 2006.

9. Na IK, Scheibenbogen C, Adam C, Stroux A, Ghadjar P, Thiel E, Keilholz U and Coupland SE: Nuclear expression of CXCR4 in tumor cells of non-small cell lung cancer is correlated with lymph node metastasis. Hum Pathol 39: 1751-1755, 2008.

10. Maekawa S, Iwasaki A, Shirakusa T, Enatsu S, Kawakami T, Kuroki M and Kuroki M: Correlation between lymph node metastasis and the expression of VEGF-C, VEGF-D and VEGFR-3 in T1 lung adenocarcinoma. Anticancer Res 27: 3735-3741, 2007. 
11. Li J, Li BL, Zhang HQ, Xu SF, Liu ZD, Yue WT and Han Y: Relationship between vascular endothelial growth factor $\mathrm{C}$ expression level and lymph node metastasis in non small cell lung cancer. Zhonghua Yi Xue Za Zhi 88: 2982-2985, 2008.

12. Kikuchi T, Daigo Y, Katagiri T, Tsunoda T, Okada K, Kakiuchi S, Zembutsu H, Furukawa Y, Kawamura M, Kobayashi K, et al: Expression profiles of non-small cell lung cancers on cDNA microarrays: Identification of genes for prediction of lymph-node metastasis and sensitivity to anti-cancer drugs. Oncogene 22 : 2192-2205, 2003.

13. Li L, Lei Q, Zhang S, Kong L and Qin B: Screening and identification of key biomarkers in hepatocellular carcinoma: Evidence from bioinformatic analysis. Oncol Rep 38: 2607-2618, 2017.

14. Kan T, Shimada Y, Sato F, Ito T, Kondo K, Watanabe G, Maeda M, Yamasaki S, Meltzer SJ and Imamura M: Prediction of lymph node metastasis with use of artificial neural networks based on gene expression profiles in esophageal squamous cell carcinoma. Ann Surg Oncol 11: 1070-1078, 2004.

15. O'Donnell RK, Kupferman M, Wei SJ, Singhal S, Weber R, O'Malley B, Cheng Y, Putt M, Feldman M, Ziober B and Muschel RJ: Gene expression signature predicts lymphatic metastasis in squamous cell carcinoma of the oral cavity. Oncogene 24: 1244-1251, 2005.

16. Nguyen ST, Hasegawa S, Tsuda H, Tomioka H, Ushijima M, Noda M, Omura K and Miki Y: Identification of a predictive gene expression signature of cervical lymph node metastasis in oral squamous cell carcinoma. Cancer Sci 98: 740-746, 2007.

17. Kim TJ, Choi JJ, Kim WY, Choi CH, Lee JW, Bae DS, Son DS, Kim J, Park BK, Ahn G, et al: Gene expression profiling for the prediction of lymph node metastasis in patients with cervical cancer. Cancer Sci 99: 31-38, 2008.

18. Edgar R, Domrachev M and Lash AE: Gene expression omnibus: NCBI gene expression and hybridization array data repository Nucleic Acids Res 30: 207-210, 2002.

19. Wright CM, Savarimuthu Francis SM, Tan ME, Martins MU, Winterford C, Davidson MR, Duhig EE, Clarke BE, Hayward NK, Yang IA, et al: MS4A1 dysregulation in asbestos-related lung squamous cell carcinoma is due to CD20 stromal lymphocyte expression. PLoS One 7: e34943, 2012.

20. Tomida S, Takeuchi T, Shimada Y, Arima C, Matsuo K, Mitsudomi T, Yatabe Y and Takahashi T: Relapse-related molecular signature in lung adenocarcinomas identifies patients with dismal prognosis. J Clin Oncol 27: 2793-2799, 2009

21. Barrett T, Wilhite SE, Ledoux P, Evangelista C, Kim IF, Tomashevsky M, Marshall KA, Phillippy KH, Sherman PM, Holko M, et al: NCBI GEO: Archive for functional genomics data sets - update. Nucleic Acids Res 41(Database issue): D991-D995, 2013.

22. Huang DW, Sherman BT, Tan Q, Collins JR, Alvord WG, Roayaei J, Stephens R, Baseler MW, Lane HC and Lempicki RA: The DAVID gene functional classification tool: A novel biological module-centric algorithm to functionally analyze large gene lists. Genome Biol 8: R183, 2007.

23. Ashburner M, Ball CA, Blake JA, Botstein D, Butler $\mathrm{H}$ Cherry JM, Davis AP, Dolinski K, Dwight SS, Eppig JT, et al: Gene ontology: Tool for the unification of biology. The gene ontology consortium. Nat Genet 25: 25-29, 2000.

24. Kanehisa M: The KEGG database. Novartis Found Symp 247: 91-101,2002

25. Ni M, Liu X, Wu J, Zhang D, Tian J, Wang T, Liu S, Meng Z, Wang K, Duan X, et al: Identification of candidate biomarkers correlated with the pathogenesis and prognosis of non-small cell lung cancer via integrated bioinformatics analysis. Front Genet 9: 469, 2018.

26. Szklarczyk D, Franceschini A, Wyder S, Forslund K, Heller D, Huerta-Cepas J, Simonovic M, Roth A, Santos A, Tsafou KP, et al: STRING v10: Protein-protein interaction networks, integrated over the tree of life. Nucleic Acids Res 43(Database issue): D447-D452, 2015.

27. Smoot ME, Ono K, Ruscheinski J, Wang PL and Ideker T: Cytoscape 2.8: New features for data integration and network visualization. Bioinformatics 27: 431-432, 2011.

28. Huang HM, Jiang X, Hao ML, Shan MJ, Qiu Y, Hu GF, Wang Q, Yu ZQ, Meng LB and Zou YY: Identification of biomarkers in macrophages of atherosclerosis by microarray analysis. Lipids Health Dis 18: 107, 2019.

29. Bader GD and Hogue CW: An automated method for finding molecular complexes in large protein interaction networks. BMC Bioinformatics 4: 2, 2003.
30. Cerami E, Gao J, Dogrusoz U, Gross BE, Sumer SO, Aksoy BA, Jacobsen A, Byrne CJ, Heuer ML, Larsson E, et al: The cBio cancer genomics portal: An open platform for exploring multidimensional cancer genomics data. Cancer Discov 2: 401-404, 2012.

31. Maere S, Heymans K and Kuiper M: BiNGO: A cytoscape plugin to assess overrepresentation of gene ontology categories in biological networks. Bioinformatics 21: 3448-3449, 2005.

32. Nagy Á, Lánczky A, Menyhárt O and Győrffy B: Validation of miRNA prognostic power in hepatocellular carcinoma using expression data of independent datasets. Sci Rep 8: 9227, 2018.

33. Siegel R, Ma J, Zou Z and Jemal A: Cancer statistics, 2014. CA Cancer J Clin 64: 9-29, 2014.

34. Detterbeck FC, Boffa DJ and Tanoue LT: The new lung cancer staging system. Chest 136: 260-271, 2009.

35. Suntharalingam M, Paulus R, Edelman MJ, Krasna M, Burrows W, Gore E, Wilson LD and Choy H: Radiation therapy oncology group protocol 02-29: A phase II trial of neoadjuvant therapy with concurrent chemotherapy and full-dose radiation therapy followed by surgical resection and consolidative therapy for locally advanced non-small cell carcinoma of the lung. Int J Radiat Oncol Biol Phys 84: 456-463, 2012.

36. Shim SS, Lee KS, Kim BT, Chung MJ, Lee EJ, Han J, Choi JY, Kwon OJ, Shim YM and Kim S: Non-small cell lung cancer: Prospective comparison of integrated FDG PET/CT and CT alone for preoperative staging. Radiology 236: 1011-1019, 2005.

37. McLoud TC, Bourgouin PM, Greenberg RW, Kosiuk JP, Templeton PA, Shepard JA, Moore EH, Wain JC, Mathisen DJ and Grillo HC: Bronchogenic carcinoma: Analysis of staging in the mediastinum with CT by correlative lymph node mapping and sampling. Radiology 182: 319-323, 1992.

38. Toloza EM, Harpole L and McCrory DC: Noninvasive staging of non-small cell lung cancer: A review of the current evidence. Chest 123(1 Suppl): 137S-146S, 2003.

39. Takamochi K, Yoshida J, Murakami K, Niho S, Ishii G, Nishimura M, Nishiwaki Y, Suzuki K and Nagai K: Pitfalls in lymph node staging with positron emission tomography in non-small cell lung cancer patients. Lung Cancer 47: 235-242, 2005.

40. Billé A, Pelosi E, Skanjeti A, Arena V, Errico L, Borasio P, Mancini M and Ardissone F: Preoperative intrathoracic lymph node staging in patients with non-small-cell lung cancer: Accuracy of integrated positron emission tomography and computed tomography. Eur J Cardiothorac Surg 36: 440-445, 2009.

41. Billè A, Okiror L, Skanjeti A, Errico L, Arena V, Penna D, Ardissone F and Pelosi E: Evaluation of integrated positron emission tomography and computed tomography accuracy in detecting lymph node metastasis in patients with adenocarcinoma vs. squamous cell carcinoma. Eur J Cardiothorac Surg 43: 574-579, 2013.

42. Pavlyk I, Leu NA, Vedula P, Kurosaka S and Kashina A: Rapid and dynamic arginylation of the leading edge $\beta$-actin is required for cell migration. Traffic 19: 263-272, 2018.

43. Lim YH, Burke AB, Roberts MS, Collins MT and Choate KA Multilineage ACTB mutation in a patient with fibro-osseous maxillary lesion and pilocytic astrocytoma. Am J Med Genet A 176: 2037-2040, 2018

44. Antonescu CR, Agaram NP, Sung YS, Zhang L, Swanson D and Dickson BC: A distinct malignant epithelioid neoplasm with GLI1 gene rearrangements, frequent S100 protein expression, and metastatic potential: Expanding the spectrum of pathologic entities with ACTB/MALAT1/PTCH1-GLI1 fusions. Am J Surg Pathol 42: 553-560, 2018

45. Castro E, Cortes-Santiago N, Ferguson LM, Rao PH, Venkatramani R and López-Terrada D: Translocation $\mathrm{t}(7 ; 12)$ as the sole chromosomal abnormality resulting in ACTB-GLI fusion in pediatric gastric pericytoma. Hum Pathol 53: 137-141, 2016.

46. Huang R and Rofstad EK: Integrins as therapeutic targets in the organ-specific metastasis of human malignant melanoma. J Exp Clin Cancer Res 37: 92, 2018

47. Yan M, Zhang L, Li G, Xiao S, Dai J and Cen X: Long noncoding RNA linc-ITGB1 promotes cell migration and invasion in human breast cancer. Biotechnol Appl Biochem 64: 5-13, 2017.

48. Wang L, Zhang Y, Lv W, Lu J, Mu J, Liu Y and Dong P: Long non-coding RNA Linc-ITGB1 knockdown inhibits cell migration and invasion in GBC-SD/M and GBC-SD gallbladder cancer cell lines. Chem Biol Drug Des 86: 1064-1071, 2015. 
49. Klahan S, Huang WC, Chang CM, Wong HS, Huang CC, Wu MS, Lin YC, Lu HF, Hou MF and Chang WC: Gene expression profiling combined with functional analysis identify integrin beta1 (ITGB1) as a potential prognosis biomarker in triple negative breast cancer. Pharmacol Res 104: 31-37, 2016.

50. Wang XM, Li J, Yan MX, Liu L, Jia DS, Geng Q, Lin HC, $\mathrm{He} \mathrm{XH}, \mathrm{Li} \mathrm{JJ}$ and Yao M: Integrative analyses identify osteopontin, LAMB3 and ITGB1 as critical pro-metastatic genes for lung cancer. PLoS One 8: e55714, 2013.

51. Qin Q, Wei F, Zhang J and Li B: miR-134 suppresses the migration and invasion of nonsmall cell lung cancer by targeting ITGB1. Oncol Rep 37: 823-830, 2017.

52. Demin DE, Bogolyubova AV, Zlenko DV, Uvarova AN, Deikin AV, Putlyaeva LV, Belousov PV, Mitkin NA, Korneev KV, Sviryaeva EN, et al: The novel short isoform of securin stimulates the expression of cyclin D3 and angiogenesis factors VEGFA and FGF2, but does not affect the expression of MYC transcription factor. Mol Biol (Mosk) 52: 508-518, 2018.

53. Zaoui K, Benseddik K, Daou P, Salaün D and Badache A: ErbB2 receptor controls microtubule capture by recruiting ACF7 to the plasma membrane of migrating cells. Proc Natl Acad Sci USA 107: 18517-18522, 2010.

54. Huang CC, Kuo HM, Wu PC, Cheng SH, Chang TT, Chang YC, Kung ML, Wu DC, Chuang JH and Tai MH Soluble delta-like 1 homolog (DLK1) stimulates angiogenesis through Notch1/Akt/eNOS signaling in endothelial cells. Angiogenesis 21: 299-312, 2018.

55. Joassard OR, Amirouche A, Gallot YS, Desgeorges MM, Castells J, Durieux AC, Berthon P and Freyssenet DG: Regulation of Akt-mTOR, ubiquitin-proteasome and autophagy-lysosome pathways in response to formoterol administration in rat skeletal muscle. Int J Biochem Cell Biol 45: 2444-2455, 2013.
56. Inoue J, Ninomiya $M$, Umetsu $T$, Nakamura $T$, Kogure $T$, Kakazu E, Iwata T, Takai S, Sano A, Fukuda M, et al: Small interfering RNA screening for the small GTPase rab proteins identifies Rab5B as a major regulator of hepatitis B virus production. J Virol 93: e00621, 2019.

57. Yamada K, Nakayama M, Miura Y, Nakano H, Mimura N and Yoshida S: Role of AVP in the regulation of vascular tonus and blood pressure in patients with chronic renal failure. Regul Pept 45: 91-95, 1993

58. Du Q, Guo X, Zhang X, Zhou W, Liu Z, Wang J, Zhang T, Mao Z, Luo J, Jin T and Liu C: SYNJ2 variant rs9365723 is associated with colorectal cancer risk in Chinese Han population. Int J Biol Markers 31: e138-e143, 2016.

59. Nakatsu F, Perera RM, Lucast L, Zoncu R, Domin J, Gertler FB, Toomre D and De Camilli P: The inositol 5-phosphatase SHIP2 regulates endocytic clathrin-coated pit dynamics. J Cell Biol 190: 307-315, 2010.

60. Dasgupta S, Jang JS, Shao C, Mukhopadhyay ND, Sokhi UK, Das SK, Brait M, Talbot C, Yung RC, Begum S, et al: SH3GL2 is frequently deleted in non-small cell lung cancer and downregulates tumor growth by modulating EGFR signaling. J Mol Med (Berl) 91: 381-393, 2013.

61. Caceres PS, Mendez M and Ortiz PA: Vesicle-associated membrane protein 2 (VAMP2) but not VAMP3 mediates cAMP-stimulated trafficking of the renal Na+-K+-2Cl- co-transporter NKCC2 in thick ascending limbs. J Biol Chem 289: 23951-23962, 2014.

(i) $(9$ This work is licensed under a Creative Commons Attribution-NonCommercial-NoDerivatives 4.0 International (CC BY-NC-ND 4.0) License. 